\title{
A VANISHING THEOREM FOR OPEN ORBITS ON COMPLEX FLAG MANIFOLDS
}

\author{
WILFRIED SCHMID ${ }^{1}$ AND JOSEPH A. WOLF ${ }^{2}$
}

\begin{abstract}
A real reductive Lie group $G$ acts on complex flag manifolds $G_{\mathbf{C}}$ /(parabolic subgroup). The open orbits $D=G(x)$ are precisely the homogeneous complex manifolds $G / H$, where $H$ is the centralizer of a torus. We prove that $D$ is $(s+1)$-complete in the sense of Andreotti and Grauert, with $s=$ complex dimension of a maximal compact subvariety of $D$. Thus $H^{q}(D, \mathcal{F})=0$ for $q>s$ and any coherent sheaf $\mathcal{F} \rightarrow D$. This vanishing theorem is needed for the realization of certain unitary representations on Dolbeault cohomology groups of homogeneous vector bundles.
\end{abstract}

Real group orbits on complex flag manifolds are important in several parts of mathematics, especially in group representation theory. See $[\mathbf{2}-\mathbf{5}]$, which rely on special cases of the following useful result.

THEOREM. Let $G$ be a connected reductive real Lie group. Let $D$ be an open $G$-orbit on a complex flag manifold $X=G_{\mathbf{C}} / Q$. Let $Y$ be a maximal compact subvariety of $D$ and $s=\operatorname{dim}_{\mathrm{C}} Y$. Then $D$ is $(s+1)$-complete in the sense of Andreotti and Grauert [1]. In particular, if $\mathcal{F} \rightarrow D$ is a coherent analytic sheaf and if $q>s$, then $H^{q}(D ; \mathcal{F})=0$.

REMARK. The argument is a variation on the proof of the special case [3, Theorem 4.1].

ProOF. We may, and do, assume $G_{\mathbf{C}}$ simply connected and semisimple, and $G \subset G_{\mathbf{C}}$. Now $D=G / H$, where $H=G \cap Q, \mathfrak{q}_{ \pm}$represent the holomorphic and antiholomorphic tangent spaces of $D$ at $1 \cdot H$, and $Q$ is the parabolic subgroup of $G_{\mathbf{C}}$ with Lie algebra $\mathfrak{h}+\mathfrak{q}_{-}$. Set $\mathfrak{q}=\mathfrak{q}_{+}+\mathfrak{q}_{-}$so $\mathfrak{g}=\mathfrak{h}+\mathfrak{q}$.

The real Lie algebra $\mathfrak{h}_{0}$ of $H$ contains a fundamental Cartan subalgebra $\mathfrak{b}_{0}$ of $\mathfrak{g}_{0}$ stable under a Cartan involution $\theta$ of $\mathfrak{g}_{0}$. Decompose $\mathfrak{g}_{0}=\mathfrak{k}_{0}+\mp_{0}$ and $\mathfrak{b}_{0}=\mathfrak{t}_{0}+\mathfrak{a}_{0}$ under $\theta$.

The fact that $D$ is open in $X$ is equivalent to $\mathfrak{q}_{-}+\overline{\mathfrak{q}_{+}}$, conjugate of $\mathfrak{g}$ over $\mathfrak{g}_{0}$. Write $\Phi(\cdot)$ for the set of $\mathfrak{b}$-roots in the indicated subspace. The parabolic $\mathfrak{h}+\mathfrak{q}_{+}$ being $\theta$-stable we have $\nu \in \mathfrak{b}^{*}$ in the weight lattice, so that

$$
\nu \perp \Phi(\mathfrak{h}) \text { and }(\nu, \alpha)>0 \text { for all } \alpha \in \Phi\left(\mathfrak{q}_{+}\right) .
$$

Now set

$$
\lambda=\nu+\theta(\nu): \quad \lambda \perp \Phi(\mathfrak{h}),(\lambda, \alpha)>0 \text { for } \alpha \in \Phi\left(\mathfrak{q}_{+}\right), \lambda(\mathfrak{a})=0 .
$$

Received by the editors July 29, 1983 .

1980 Mathematics Subject Classification. Primary 22E46, 32F10; Secondary 53C55.

1 Partially supported by NSF Grant MCS-13190.

${ }^{2}$ Partially supported by NSF Grant MCS- 8200235 . 
Then $\lambda$ exponentiates to a unitary character $e^{\lambda} \in \hat{H}$. Consider the hermitian holomorphic line bundle

$$
\mathbf{L}_{\lambda} \rightarrow G / H \text { associated to } G \rightarrow G / H \text { by } e^{\lambda} .
$$

The metric connection for its $G$-invariant hermitian metric $h_{0}$ has connection form $\lambda$ (extended to $\mathfrak{g}$ by zero on all root spaces) and curvature form $\omega_{0}$. These are related by

$$
\omega_{0}=2 \pi \sqrt{-1} d \lambda \quad \text { and } \quad \omega_{0}=-\partial \bar{\partial} \log h_{0} .
$$

If $\alpha, \beta \in \Phi\left(\mathfrak{q}_{+}\right)$, then

if $\bar{\beta} \neq-\alpha: \lambda\left(\left[e_{\alpha}, \bar{e}_{\beta}\right]\right)=0$ and $\left\langle e_{\alpha}, \bar{e}_{\beta}\right\rangle=0$,

if $\bar{\beta}=-\alpha:\left[e_{\alpha}, \bar{e}_{\beta}\right]=c h_{\alpha}$ for some $c \neq 0$, and

$$
\lambda\left(\left[e_{\alpha}, \bar{e}_{\beta}\right]\right)=(\lambda, \alpha) c=(\lambda, \alpha)\left\langle e_{\alpha}, \bar{e}_{\beta}\right\rangle .
$$

Let $x, y \in \mathfrak{q}_{+}$, say $x=\sum x_{\alpha}$ and $y=\sum y_{\beta}$, where $x_{\gamma}, y_{\gamma} \in \mathfrak{g}_{\gamma}$. Then

$$
\begin{aligned}
\left(\sqrt{-1} \partial \bar{\partial} \log h_{0}\right)(x, y) & =2 \pi d \lambda(x, \bar{y})=\pi \lambda([x, \bar{y}]) \\
& =\pi \sum_{\alpha, \beta \in \Phi\left(\mathfrak{q}_{+}\right)}(\lambda, \alpha)\left\langle x_{\alpha}, \bar{y}_{\beta}\right\rangle .
\end{aligned}
$$

But each $(\lambda, \alpha)>0$, and $\langle x, \bar{y}\rangle$ is positive definite on $\mp \cap \mathfrak{q}_{+}$, negative definite on $\mathfrak{k} \cap \mathfrak{q}_{+}$. With $n=\operatorname{dim}_{\mathbf{C}} X$ and $s=\operatorname{dim}_{\mathbf{C}} Y$ we conclude that

the hermitian form $\sqrt{-1} \partial \bar{\partial} \log h_{0}$ on the holomorphic tangent bundle of $D$ has signature: $n-s$ pluses and $s$ minuses.

Note that $e^{\lambda}$ extends to a holomorphic map $Q \rightarrow \mathrm{GL}(1 ; C)$ by triviality on $\exp \left(\mathfrak{q}_{-}\right)$. Thus $\mathbf{L}_{\lambda} \rightarrow D$ extends to a homogeneous holomorphic line bundle

$$
\tilde{\mathbf{L}}_{\lambda} \rightarrow G_{\mathbf{C}} / Q \text { associated to } G_{\mathbf{C}} \rightarrow G_{\mathbf{C}} / Q \text { by } e^{\lambda} \text {. }
$$

$\mathfrak{g}$ has a compact real form $\mathfrak{g}_{u}=\mathfrak{k}_{0}+\sqrt{-1} \mp_{0}$, and $X=G_{u} / H_{u}$, where $\mathfrak{h}_{u}=\mathfrak{h} \cap \mathfrak{g}_{u}$. Note that $e^{\lambda}$ is unitary on $H_{u}$, so $\tilde{\mathbf{L}}_{\lambda}$ has a $G_{u}$-invariant hermitian metric $h_{u}$, connection form $\lambda$ and curvature form $\omega_{u}$. The difference between $h_{u}$ and $h_{0}$, and $\omega_{u}$ and $\omega_{0}$, is that we use conjugation of $\mathfrak{g}$ over $\mathfrak{g}_{u}$ rather than $\mathfrak{g}_{0}$. Denote it $x \rightarrow \overline{\bar{x}}$ $(=\theta \bar{x})$. As above,

$$
\left(\sqrt{-1} \partial \bar{\partial} \log h_{u}\right)(x, y)=\pi \sum_{\alpha, \beta \in \Phi\left(\mathfrak{q}_{+}\right)}(\lambda, \alpha)\left(x_{\alpha}, \overline{\bar{y}}_{\beta}\right)
$$

and so

the hermitian form $\sqrt{-1} \partial \bar{\partial} \log h_{u}$ on the holomorphic tangent bundle of $D$ is negative definite.

The ratio $f=h_{0} / h_{u}$ is $C^{\infty}$ and positive in $D$. We are going to show that $\phi=\log f$ is an exhaustion function for $D$ whose Levi form has at least $n-s$ positive eigenvalues at every point of $D$. Then $D$ will be $(s+1)$-complete.

Let $g \in G$. As $h_{0}$ is $G$-invariant, $\sqrt{-1} \partial \bar{\partial} \log h_{0}$ is positive definite on the $(n-s)$ dimensional subspace $\operatorname{Ad}(g)\left(\mp \cap \mathfrak{q}_{+}\right)$of the holomorphic tangent space $\operatorname{Ad}(g) \mathfrak{q}_{+}$at $g H$. Also, $\sqrt{-1} \partial \bar{\partial} \log h_{u}$ is negative definite there, because it is negative definite everywhere. Thus the Levi form

$$
L(\phi)=\sqrt{-1} \partial \bar{\partial} \log h_{0}-\sqrt{-1} \partial \bar{\partial} \log h_{u}
$$


is positive definite on $\operatorname{Ad}(g)\left(\mp \cap \mathfrak{q}_{+}\right)$. We have just verified that $L(\phi)$ has at least $n-s$ positive eigenvalues at every point of $D$.

It remains, now, only to show that $\phi=\log f$ is an exhaustion function for $D$, i.e. that

$$
\{x \in D: \phi(x) \leq c\} \quad \text { is compact for every } c \in \mathbf{R} .
$$

For that, we need only prove that $e^{-\phi}$ has a continuous extension to $X$ which vanishes on the topological boundary $\operatorname{bd}(D)$ of $D$ in $X$. For then

$$
\{x \in D: \phi(x) \leq c\}=\left\{x \in D: e^{-\phi(x)} \geq e^{-c}\right\}
$$

while $e^{-c}>0$ so that set is interior to $D \cup \operatorname{bd}(D)$, while $\left\{x \in X: e^{-\phi(x)} \geq e^{-c}\right\}$ is compact because $X$ is compact.

In fact, we need only prove that $e^{-\phi}$ has a continuous extension to $X$ that vanishes on $\operatorname{bd}(D)$ for one particular choice of $\lambda$. We will use

$$
\lambda=\rho_{G / H}+\theta\left(\rho_{G / H}\right)=2 \rho_{G / H},
$$

where $\rho_{G / H}$ is half the sum of the roots in $\Phi\left(\mathfrak{q}_{+}\right)$. Here note that

$$
\left(\rho_{G / H}, \alpha\right)=0 \quad \text { if } \alpha \in \Phi(\mathfrak{h}), \quad>0 \quad \text { if } \alpha \in \Phi\left(\mathfrak{q}_{+}\right), \quad<0 \quad \text { if } \alpha \in \Phi\left(\mathfrak{q}_{-}\right)
$$

simply because $\mathfrak{h}+\mathfrak{q}_{+}$is a parabolic subalgebra of $\mathfrak{g}$. Now, with $\lambda$ as above,

$$
\mathbf{L}_{\lambda}=\mathbf{K}_{D}^{*} \quad \text { and } \quad \tilde{\mathbf{L}}_{\lambda}=\mathbf{K}_{X}^{*} \quad \text { (dual line bundles), }
$$

where $K_{D} \rightarrow D$ and $K_{X} \rightarrow X$ are the canonical line bundles. Since $e^{-\phi}=e^{-\log f}=$ $1 / f=h_{u} / h_{0}$, and since we have

$$
\begin{aligned}
& h_{0}^{*}: G \text {-invariant hermitian metric on } \mathbf{K}_{D}, \\
& h_{u}^{*}: G_{u} \text {-invariant hermitian metric on } \mathbf{K}_{X}
\end{aligned}
$$

such that $h_{0} h_{0}^{*}=1$ on $D$ and $h_{u} h_{u}^{*}=1$ on $X$, we have $e^{-\phi}=h_{0}^{*} / h_{u}^{*}$. So we will complete the proof by showing that $h_{0}^{*} / h_{u}^{*}$ has a continuous extension to $X$ that vanishes on $\operatorname{bd}(D)$.

The holomorphic cotangent bundle $\mathbf{T}_{X}^{*} \rightarrow X$ has fibre at $g Q$ given by $\operatorname{Ad}(g) \mathfrak{q}_{+}^{*}=$ $\operatorname{Ad}(g) q_{\text {.. }}$ It has $G_{u}$-invariant hermitian metric given at $q G$ by

$$
F_{u}(\xi, \eta)=-\langle\xi, \overline{\bar{\eta}}\rangle \quad \text { for } \xi, \eta \in \operatorname{Ad}(g) \mathfrak{q}_{-} .
$$

Similarly $\mathbf{T}_{D}^{*}=\left.\mathbf{T}_{X}^{*}\right|_{D}$ has $G$-invariant indefinite-hermitian metric given at $g H$, $g \in G$, by

$$
F_{0}(\xi, \eta)=-\langle\xi, \bar{\eta}\rangle \quad \text { for } \xi, \eta \in \operatorname{Ad}(g) \mathfrak{q}_{-} .
$$

Note that, since $\mathbf{K}_{D}=\Lambda^{n} \mathbf{T}_{D}^{*}$ and $\mathbf{K}_{X}=\Lambda^{n} \mathbf{T}_{X}^{*}$, there is a real constant $c \neq 0$ such that

$$
h_{0}^{*} / h_{u}^{*}=c\left(\text { determinant of } F_{0} \text { with respect to } F_{u}\right) \text {. }
$$

Thus

$$
f^{*}(g Q)=c\left(\text { determinant of } F_{0} \text { on } \operatorname{Ad}(g) \mathfrak{q}_{-} \text {with respect to } F_{u}\right)
$$

is a $C^{\infty}$ function on $X$ that extends $h_{0}^{*} / h_{u}^{*}$ from $D$ to $X$.

It now remains only to show that $f^{*}$ vanishes on $\operatorname{bd}(D)$, i.e. that the hermitian form $F_{0}$ on $\operatorname{Ad}(g) q_{-}$is singular whenever $g Q \in \operatorname{bd}(D)$. If $g Q \in \operatorname{bd}(D)$, then its $G$-orbit on $X$ is of positive codimension, so

$$
\operatorname{Ad}(g)\left(\mathfrak{h}+\mathfrak{q}_{-}\right)+\overline{\operatorname{Ad}(g)\left(\mathfrak{h}+\mathfrak{q}_{-}\right)} \neq \mathfrak{g} .
$$


Choose a $\theta$-stable Cartan subalgebra $\tilde{\mathfrak{b}}_{0}$ of $\mathfrak{g}_{0}$ contained in $\operatorname{Ad}(g)\left(\mathfrak{h} \cap \mathfrak{q}_{-}\right)$. Then there is a $\tilde{\mathfrak{b}}$-root $\alpha$ such that $\mathfrak{g}_{\alpha} \subset \operatorname{Ad}(g) \mathfrak{q}_{-}$, but

$$
\mathfrak{g}_{-\alpha} \not \subset \operatorname{Ad}(g)\left(\mathfrak{h}+\mathfrak{q}_{-}\right)+\overline{\operatorname{Ad}(g)\left(\mathfrak{h}+\mathfrak{q}_{-}\right)}
$$

Thus, if $\beta$ is any $\tilde{\mathfrak{b}}$-root with $\mathfrak{g}_{\beta} \subset \operatorname{Ad}(g) \mathfrak{q}_{-}$we have $F_{0}\left(e_{\alpha}, e_{\beta}\right)=\left\langle e_{\alpha}, \bar{e}_{\beta}\right\rangle$, nonzero only if $\bar{\beta}=-\alpha$, while $\bar{\beta}=-\alpha$ would imply $\mathfrak{g}_{-\alpha} \subset \overline{\operatorname{Ad}(g) \mathfrak{q}_{-}}$, which is false. Thus $F_{0}$ is singular on $\operatorname{Ad}(g) \mathfrak{q}_{-}$. That completes the proof. Q.E.D.

\section{REFERENCES}

1. A. Andreotti and H. Grauert, Théorèmes de finitude pour la cohomologie des espaces complexes, Bull. Soc. Math. France 90 (1962), 193-259.

2. J. Rawnsley, W. Schmid and J. A. Wolf, Singular representations and indefinite harmonic theory, J. Funct. Anal. 51 (1983), 1-114.

3. W. Schmid, Homogeneous complex manifolds and representations of semisimple Lie groups, Thesis, University of California at Berkeley, 1967; Proc. Nat. Acad. Sci. U.S.A. 59 (1968), 56-59.

4.,$L^{2}$ cohomology and the discrete series, Ann. of Math. (2) 103 (1976), 375-394.

5. J. A. Wolf, The action of a real semisimple Lie group on a complex flag manifold. II: Unitary representations on partially holomorphic cohomology spaces, Mem. Amer. Math. Soc. No. 138 (1974).

Department of Mathematics, Harvard University, Cambridge, MassachuSETTS 02138

Department of Mathematics, University of California, Berkeley, CaliforNIA 94720 